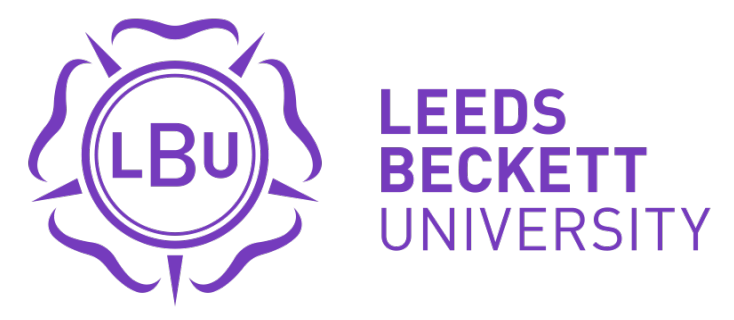

Citation:

Jones, TW and Walshe, IH and Hamilton, DL and Howatson, $\mathrm{G}$ and Russell, $\mathrm{M}$ and Price, OJ and St Clair Gibson, A and French, DN (2015) Signalling Responses Following Varying Sequencing of Strength and Endurance Training in a Fed State. International journal of sports physiology and performance. ISSN 1555-0265 DOI: https://doi.org/10.1123/ijspp.2015-0534

Link to Leeds Beckett Repository record:

https://eprints.leedsbeckett.ac.uk/id/eprint/2546/

Document Version:

Article (Updated Version)

The aim of the Leeds Beckett Repository is to provide open access to our research, as required by funder policies and permitted by publishers and copyright law.

The Leeds Beckett repository holds a wide range of publications, each of which has been checked for copyright and the relevant embargo period has been applied by the Research Services team.

We operate on a standard take-down policy. If you are the author or publisher of an output and you would like it removed from the repository, please contact us and we will investigate on a case-by-case basis.

Each thesis in the repository has been cleared where necessary by the author for third party copyright. If you would like a thesis to be removed from the repository or believe there is an issue with copyright, please contact us on openaccess@leedsbeckett.ac.uk and we will investigate on a case-by-case basis. 


\section{SIGNALLING RESPONSES FOLLOWING VARYING SEQUENCING OF STRENGTH AND ENDURANCE TRAINING IN A FED STATE}

Submission type: Original Investigation

Thomas W. Jones ${ }^{1}$, Ian H. Walshe ${ }^{2}$, David L. Hamilton ${ }^{2}$, Glyn Howatson ${ }^{3,4}$, Mark Russell ${ }^{3}$, Oliver J. Price ${ }^{3,5}$, Alan St Clair Gibson ${ }^{6}$ and Duncan N. French ${ }^{3,7}$

${ }^{1}$ ASPIRE, Academy for Sports Excellence, Doha, Qatar

${ }^{2}$ Health and Exercise Sciences Research Group, School of Sport, University of Stirling, Stirling, United Kingdom

${ }^{3}$ Department of Sport, Exercise and Rehabilitation, Northumbria University, Newcastle upon Tyne, United Kingdom

${ }^{4}$ Water Research Group, School of Environmental Sciences and Development, North West University, Potchefstroom, South Africa

${ }^{5}$ National Heart and Lung Institute, Imperial College London, London, United Kingdom

${ }^{6}$ School of Medicine, University of the Free State, Bloemfontein, South Africa

${ }^{7}$ English Institute of Sport, Sportcity, Manchester, United Kingdom

Dr Duncan N French

Department of Sport and Exercise, and Rehabilitation

Northumbria University

Northumberland Building

Newcastle upon Tyne

NE1 8ST

duncan.french@northumbria.ac.uk

Preferred Running Head: Signalling Responses to Concurrent Training Sequencing

Word count: 3359

Abstract word count: 249 


\begin{abstract}
Purpose: The objective of this study was to compare anabolic signalling responses to differing sequences of concurrent strength and endurance training in a fed state.

Methods: Eighteen resistance-trained males were randomly assigned to the following experimental conditions; i) strength training (ST), ii) strength followed by endurance training (ST-END) or iii) endurance followed by strength training (END-ST). Muscle tissue samples were taken from the vastus lateralis before each exercise protocol, upon cessation of exercise, and $1 \mathrm{~h}$-post cessation of strength training. Tissue was analysed for total and phosphorylated (p-) signalling proteins linked to the mTOR and AMPK networks.
\end{abstract}

Results: Strength training performance was similar between ST, ST-END and END-ST. pS6k1 was elevated from baseline $1 \mathrm{~h}$ post training in ST and ST-END (both $p<0.05$ ). p-4EBP1 was significantly lower than baseline post ST $(p=0.01)$, while $1 \mathrm{~h}$ post exercise in the ST-END condition $\mathrm{p}-4 \mathrm{E}-\mathrm{BP} 1 \mathrm{was}$ significantly greater than post exercise $(p=0.04)$. p-ACC was elevated from baseline both post and $1 \mathrm{~h}$ post exercise (both $p<0.05$ ) in the END-ST condition. AMPK, mTOR, p38, PKB, eEF2 responded similarly to the ST, ST-END and ENDST. Signalling responses to ST, ST-END and END were largely similar. As such it cannot be ascertained which sequence of concurrent strength and endurance training is most favourable in promoting anabolic signalling.

Conclusions: These data indicate that in the case of the present study an acute bout of concurrent training of differing sequences elicited similar responses of the AMPK and mTOR networks.

Key words: Combined exercise, mTOR, AMPK, exercise order, anabolic signalling 


\section{Introduction}

A number of research studies have reported acute and chronic resistance exercise-induced activation of the PI3k/PKB/mTORC1/S6k1/4E-BP1 growth associated signalling network ${ }^{1-4}$. The repeated activation of the "mTORC1 network", induced by progressively overloaded strength training, can result in increased cross sectional area (CSA) and contractile strength of the trained muscles ${ }^{5}$. It has been hypothesised that the endurance training-induced activation of the energy modulating AMPK signalling network may be antagonistic to the mTORC1 network and any associated strength training adaptations ${ }^{6-8}$.

The potential inhibition of intramuscular protein synthesis via endurance training mechanisms that activate AMPK remains a contentious issue in applied physiology. In murine models it is generally accepted that endogenous AMPK mediates a suppressive effect on mTORC1 $5,9,10$ activity and consequent muscle growth induced by external loading ${ }^{5,9,11}$. In humans the interactions between the growth-associated and energy modulating pathways are yet to be fully elucidated, as a number of researchers report no inhibitory effect of AMPK on mTORC1 and subsequent signalling ${ }^{12-15}$. Moreover, to date only two published studies have investigated the effects of differing the order of acute concurrent strength and endurance training on molecular and signalling responses associated with protein synthesis ${ }^{16,17}$.

Investigations of the molecular responses to acute intra-session sequencing of concurrent training have reported no effect of strength and endurance exercise order (strength followed by endurance or vice versa) on the mTORC1 network ${ }^{16,17}$. However, there are notable inconsistencies between studies, as Coffey, Jemiolo, Edge, Garnham, Trappe, Hawley 16 employed repeated high intensity sprints as the endurance stimulus rather than the more commonly used $\sim 30$ min of steady state exercise ${ }^{12-14}$. An additional confounding methodological factor is that no study included a condition involving strength training alone. As such, it cannot be accurately determined whether any inhibition of anabolic signalling was caused by the endurance exercise stimulus. Furthermore, in both of these studies the respective exercise protocols were conducted in a fasted state ${ }^{16,17}$. Conducting strength and/or endurance type exercise when fasted is associated with cortisol catabolising protein and phosphorylation of the AMPK network ${ }^{18}$. Furthermore, it has been suggested that low muscle glycogen may impair intracellular signalling pathways responsible for hypertrophy ${ }^{19}$. This may indicate data presented in previous research examining the signalling responses to concurrent training in which participants are fasted may not provide an accurate representation of anabolic signalling. In addition, the real world applications of these studies are lacking, as few athletes perform strength training fasted

The molecular responses to concurrent strength and endurance training in humans remain inconclusive, and thus it is difficult to fully elucidate the specific mechanisms regulating adaptations to concurrent training strategies. The purpose of this study was to answer two questions. Firstly, does combining acute bouts of strength and endurance training result in the inhibition of signalling proteins associated with hypertrophy as a result of the activation of the AMPK signalling network? Secondly, does the order in which strength and endurance training are performed influence the responses of the mTORC1 and AMPK signalling networks in a fed state?

\section{Methods}


Eighteen recreationally resistance-trained men (age: $24 \pm 3$ y; body mass: $80.5 \pm 9.9 \mathrm{~kg}$; height: $177.8 \pm 7.5 \mathrm{~cm}$; \% body fat: $17.5 \pm 7.2 \%$; sum of assessed 1 repetition maximums (leg extension and leg press) (1RMs): $375.6 \pm 56.3 \mathrm{~kg} ; \dot{V} O_{2 \max }: 50.1 \pm 7.2 \mathrm{ml} \cdot \mathrm{kg} \cdot \mathrm{min}^{-1}$ ) volunteered to participate in the study. Prior to all procedures written informed consent was given in accordance with the Declaration of Helsinki. Participants were matched at baseline for age, body mass, body fat \%, $1 \mathrm{RM}$ totals and $\dot{V} O_{2 \max }$ (all $p>0.05$ ) and randomly assigned to one experimental condition. Each participant had completed $>2$ years of strength training prior to the study. All participants were free from any endocrine or metabolic contraindications and in all cases participants were asked to refrain from nutritional supplementation or pharmacological interventions for 30 days prior to testing.

\section{Design}

A balanced, randomised, between-group study design was employed. Participants were randomly assigned to one of three experimental conditions: i) strength training (ST), ii) concurrent training, with strength training first (ST-END) or iii) concurrent training, with endurance training first (END-ST). Participants in the ST group performed strength training alone; the ST-END group performed strength training immediately followed by an endurance training protocol; those participants designated END-ST performed endurance training immediately followed by strength training. In order to assess the effect of each intervention on signalling factors related to morphological adaptation, muscle tissue samples were collected before, $10 \mathrm{~min}$ post exercise and $1 \mathrm{~h}$ post cessation of the loading protocols. The participants' ability to maintain their designated strength-training load was assessed to determine if endurance exercise prior to strength training resulted in diminished strength performance.

\section{Methodology}

Prior to any trials, assessment of $\dot{V} O_{2 \max }$ and 1RM loads were performed for the purpose of normalising relative training intensity, all assessments were conducted in line with standardised procedures ${ }^{20,21}$. Upon commencing the study, participants attended the laboratory in a fed state. Final nutritional intake was standardised prior to the experimental protocol (Figure 1), it was consumed $\geq 1 \mathrm{~h}$ prior to any loading and consisted of $2 \mathrm{~g}$ carbohydrate $/ \mathrm{kg}$ body mass, 0.5 $\mathrm{g}$ protein $/ \mathrm{kg}$ (milk protein) body mass and $0.15 \mathrm{~g}$ fat $/ \mathrm{kg}$ body mass. Participants were advised to abstain from exercise, alcohol and caffeine for $24 \mathrm{~h}$ prior to each visit.

The strength training protocol consisted of seated leg extensions and seated leg press as these exercises have previously been demonstrated to activate the vastus lateralis (VL) and have previously been employed in comparable research ${ }^{16,22}$. It was critical that the VL was activated consequent to the strength training protocol as this muscle was used for harvesting tissue. For each exercise within the strength-training bout, 5 sets of 6 repetitions at $80 \%$ 1RM were completed. This protocol and intensity of exercise has been shown to be appropriate for eliciting strength and hypertrophic responses in recreationally trained non-athletes ${ }^{23,24}$.

\section{Figure 1 about here}

In all instances, the endurance exercise protocol involved participants completing $30 \mathrm{~min}$ of submaximal cycle ergometry at $70 \%$ power at maximal oxygen uptake ( $\mathrm{p} \dot{V} O_{2 \max }$ ). Visual feedback for pedal frequency, power output and elapsed time were provided. All strength 
and/or endurance based exercise commenced at the same time of day (0900 $\mathrm{h} \pm 1 \mathrm{~h})$ to avoid any diurnal performance or signalling variations ${ }^{25}$.

Muscle biopsies were taken from the VL at baseline, $10 \mathrm{~min}$ post and $1 \mathrm{~h}$ post cessation of the loading protocols. All muscle tissue was extracted via the puncture biopsy technique from the VL. Prior to incision, local anaesthetic (Bupivacaine Hydrochloride, 0.5\% Marcaine) was injected into biopsy site. Initially $1 \mathrm{ml}$ was injected, and if a visible raise in the subcutaneous volume did not appear the needle was slightly retracted and an additional $1 \mathrm{ml}$ injected. The needle was then removed and reinserted into the injection location at $45^{\circ}$ and a further $1 \mathrm{ml}$ injected. Following a $\geq 3$ min period, an incision was made longitudinally to the line of the VL to cut through subcutaneous tissue and fascia. Following insertion the biopsy needle (Bard Biopsy Systems, Tempe, AZ, USA) was then inserted perpendicular to skin surface. When the tip of the needle passed the muscle facia the angle of the needle was flattened to $\sim 45^{\circ}$, fired, and then immediately withdrawn. The extracted tissue was immediately removed, cleaned with saline, weighed and snap frozen in liquid nitrogen before storage at $-80^{\circ} \mathrm{c}$. This process was repeated until sufficient tissue $(40-60 \mu \mathrm{g})$ was obtained for biochemical analysis.

Processed muscle tissue was analysed for total and phosphorylated signalling proteins associated with the mTOR and AMPK signalling networks. The analysed signalling proteins within the mTOR network included; 4E binding protein 1 (4E-BP1), mammalian target of rapamycin (mTOR), protein kinase $\mathrm{B}(\mathrm{PKB})$ and $70-\mathrm{kDa}$ S6 protein kinase (S6k1). The analysed signalling proteins of the AMPK network included; acetyl-CoA carboxylase (ACC), AMPK, eukaryotic elongation factor 2 (eEF2) and tuberous sclerosis complex 2 (TSC2), the stress activated protein mitogen-activated protein kinase (p38) was also analysed. Muscle

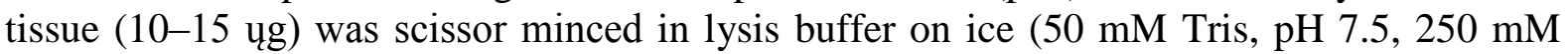
sucrose, $1 \mathrm{mM}$ EDTA, $1 \mathrm{mM}$ EGTA, $1 \%$ Triton X-100, $1 \mathrm{mM} \mathrm{NaVO4,} 50 \mathrm{mM} \mathrm{NaF}, 0.50 \%$ protease inhibitor cocktail). Samples were then shaken for $1 \mathrm{~h}(800 \mathrm{rpm})$ at $4^{\circ} \mathrm{C}$ before centrifugation for $60 \mathrm{~min}$ at $12000 \mathrm{~g}$. The supernatant was subsequently removed from the pellet to a clean tube and used to determine protein concentration via a DC Protein Assay (BioRad Laboratories, Hertfordshire, UK). Equal amounts of protein were first boiled in Laemmli sample buffer (250 mM Tris-HCl, pH 6.8, 2\% SDS, 10\% glycerol, 0.01\% bromophenol blue, $5 \% \beta$-mercaptoethanol). Subsequently $10-30 \mu$ g protein from each sample was separated on precast Criterion (Bio-Rad Laboratories) SDS polyacrylamide gels (4-20\% gradient gels) for $\sim 75 \mathrm{~min}$ at $150 \mathrm{~V}$. Proteins were transferred to a Protran nitrocellulose membrane (Whatman, Dassel, Germany) at $30 \mathrm{~V}$ for $4 \mathrm{~h}$. Membranes were then blocked in 5\% BSA-Tris-buffered saline with $0.1 \%$ Tween-20 (TBST) and then incubated overnight at $4{ }^{\circ} \mathrm{C}$ with the appropriate primary antibody. The antibodies were used at the following dilutions: rabbit monoclonal GAPDH 1:5,000, rabbit polyclonal 1:1,000 4E-BP1 ${ }^{\text {ser37/41 }}, \mathrm{ACC}^{\mathrm{ser} 79}, \mathrm{AMPK}^{\text {Thr172 }}$, eEF2 ${ }^{\text {Thr56, }}$, mTOR $^{\text {ser2448 }}, \mathrm{p}^{3} 8^{\text {Thr80/Thy182 }}, \mathrm{PKB}^{\text {ser437}}, \mathrm{S}_{\mathrm{k}} 1^{\text {Thr389 }}$ and $\mathrm{TSC}^{\mathrm{ser} 939}$ (14C10; Cell Signaling Technology, Danvers, MA).

Following overnight incubation, the membranes underwent $3 \times 5$ min washes in TBST. The membrane was then incubated for $1 \mathrm{~h}$ at room temperature with horseradish peroxidase-linked anti-rabbit IgG (1:10,000; Abcam, Cambridge, UK) or anti-mouse (1:10,000; Abcam, Cambridge, UK), diluted in 5\% BSA-TBST. The membrane was then cleared of the antibody using TBST. Antibody binding was detected using enhanced chemiluminescence (GE Healthcare Biosciences, Pittsburgh, PA). Molecular weight was estimated using molecular weight Kaleidoscope Prestained Standards (Bio-Rad Laboratories). To improve antibody performance, reduce nonspecific bands and the variability of quantifying different membranes the following procedure was performed: prior to transfer, the gels were cut at the molecular 
weight markers which corresponded to the molecular weight $(\mathrm{KDa})$ of the proteins analysed in the respective runs. All of the gel segments for the entire data set were transferred onto a single membrane for each protein. This allowed clearer visualization of the time course response of the proteins. Following imaging and band quantification of phosphorylation of the analysed proteins membranes were stripped for $30 \mathrm{~min}$ at $50^{\circ} \mathrm{C}$ in stripping buffer $(65 \mathrm{mM}$ Tris $\mathrm{HCl}, 2 \%$ SDS vol/vol, $0.8 \%$ mercaptoethanol $\mathrm{vol} / \mathrm{vol}$ ) and reblocked, followed by an overnight incubation in the corresponding total primary antibody. All imaging and band quantification were carried out using a bioimaging Gel Doc system (Bio-Rad Laboratories). Quantified phosphorylated 4E-BP1 ${ }^{\text {ser37/41 }}, \mathrm{ACC}^{\text {ser79 }}, \mathrm{AMPK}^{\text {Thr172 }}, \mathrm{eEF}^{\text {Thr56 }}, \mathrm{mTOR}^{\mathrm{ser} 2448}, \mathrm{p}^{3} 8^{\text {Thr80/Thy182 }}$, $\mathrm{PKB}^{\mathrm{ser} 437}, \mathrm{~S}_{6 \mathrm{k}} 1^{\mathrm{Thr} 389}$ and TSC2 ${ }^{\mathrm{ser} 939}$ were divided by the total corresponding protein.

\section{Statistical Analysis}

Performance data presented as mean \pm standard deviation and molecular data are presented as mean \pm standard error. Molecular and signalling data were transformed to arbitrary units and normalised to individual baseline data ${ }^{12,16}$. Prior to analysis dependant variables were verified as meeting required assumptions of parametric statistics and changes in molecular variables were analysed using mixed model repeated measures ANOVA tests. ANOVA analysed differences between 3 conditions (ST, ST-END and END-ST) and 3 time points (pre, post and $1 \mathrm{~h}$ post exercise cessation). Participant's ability to maintain their individual required training intensity were analysed using one way ANOVA tests. ANOVA analysed differences between 3 conditions ST, ST-END and END-ST). The alpha level of 0.05 was set prior to data analysis. Assumptions of sphericity were assessed using Mauchly's test of sphericity, if the assumption of sphericity was violated Greenhouse Gessier correction was employed. If significant effects between conditions or over time were observed post-hoc differences were analysed with the use of Bonferroni correction. Statistical power of the study was calculated post-hoc using G*Power statistical software (v3.1.3, Düsseldorf, Germany) using the effect size, group mean, SD and sample size of the primary outcome measures, in this case being the signalling proteins of the mTOR network. Power was calculated as between 0.8 and 1 indicating sufficient statistical power ${ }^{26}$.

\section{Results}

Participants' ability to maintain strength training load was not affected by experimental condition $\left(F_{(2,15)}=0.491, p=0.621\right.$; Figure 2$)$.

\section{Figure 2 about here}

No time $\mathrm{x}$ group interaction was observed for $\mathrm{p}-4 \mathrm{E}-\mathrm{BP} 1\left(F_{(4,28)}=0.405, p=0.804\right)$. $\mathrm{p}-4 \mathrm{E}-\mathrm{BP} 1$ changed significantly over time $\left(F_{(2,28)}=4.943, p=0.015\right)$. p-4E-BP1 was significantly lower than baseline values post exercise in participants following in ST $(29.6 \pm 13.6 \%)(p=0.01) .1$ $\mathrm{h}$ post exercise in ST-END p-4E-BP1 was $41.1 \pm 7.2 \%$ greater than post exercise $(p=0.04)$ although no difference was observed between pre and post exercise $(P=0.10)$. No differences were observed in END-ST (both $p>0.05$ ).

No time $\mathrm{x}$ group interactions were observed for p-S6k1 $\left(F_{(4,28)}=0.638, p=0.64\right)$. A time effect was observed for p-S6k1 $\left(F_{(2,28)}=11.733, p<0.001\right)$. Both ST and ST-END elicited increases from baseline (18.5 $\pm 58.7 \%$ and $57.9 \pm 93.9 \%$ respectively) in p-S6k1 $1 \mathrm{~h}$ post exercise (113.4 $\pm 119.3 \%$ and $145.6 \pm 191.4 \%$; both $p<0.05)$. No such increase from baseline was observed 
$1 \mathrm{~h}$ post exercise in END-ST $(p=0.19)$. END-ST did however elicit significant increases in $\mathrm{p}-$ S6k1 from post to $1 \mathrm{~h}$ post exercise $(p=0.04)$.

\section{Figure 3 about here}

No time x group interactions were observed for p-ACC $\left(F_{(4,28)}=1.884, p=0.141\right)$, however phosphorylation changed over time $\left(F_{(2,28)}=5.751, p=0.008\right.$; Figure 3$)$. END-ST resulted in significant elevations from baseline both post $(91.2 \pm 22.4 \%)$ and $1 \mathrm{~h}$ post exercise $(51.5 \pm$ $33.9 \%$; both $p<0.05$ ).

\section{Figure 4 about here}

Figure 5 about here

Neither time $\mathrm{x}$ group interactions $\left(F_{(4,28)}=0.873, p=0.492\right)$, nor effects of time $\left(F_{(2,28)}=\right.$ 2.494, $p=0.101$ ) were reported for p-mTOR, p-PKB, p-AMPK, p-eEF2, p-p38, p-TSC2 (Figures 3 and 4).

\section{Discussion}

The aim of this study was to examine whether manipulating the order of acute loadings of strength and endurance training influenced the responses of the mTOR and AMPK signalling networks in a fed state.

The responses of the mTOR and AMPK networks to ST, ST-END and END-ST were similar. As such, the findings of this study indicate that when strength and endurance training are performed in close proximity (following a feeding strategy) the order of the respective exercise modalities does not influence the anabolic nor energy modulating signalling responses. Similar to the present study, previous research has also reported elevations in p-S6k1 following both strength training in isolation and concurrent training ${ }^{15-17,27,28}$. The similar increases in p-S6k1 following strength training and strength training closely followed by endurance training are consistent with recent comparable research ${ }^{14}$. Additionally, Coffey et al. ${ }^{16}$ also reported upregulation of S6k1 activity following strength training, however no such increases were observed when strength training was performed following a bout of high intensity interval training. It was suggested that strength training performed after repeated sprints was undertaken in the presence of greater metabolic acidosis (confirmed by greater elevated blood lactate concentrations) when compared with the initial exercise bout, which contributed to the attenuated increase in S6k1. This hypothesis is supported by research demonstrating associations between metabolic acidosis and protein degradation in both rodents and humans 29,30 .

Unlike much other research investigating signalling proteins in response to concurrent strength and endurance training ${ }^{15-17}$, the present study was conducted following a standardised feeding strategy. This was designed to replicate conditions in which strength training would typically be conducted (i.e. real life practical application). Furthermore, low glycogen content has been shown to blunt signalling responses consequent to strength training ${ }^{31}$. It should be noted that one study has observed rates of myofibrillar protein synthesis to be similar when resistance training was conducted in the presence of high and low nutrient availability ${ }^{32}$, although this study involved resistance training in isolation. As previously stated, contrasting findings to the present study have been reported by Lundberg et al. ${ }^{27}$, who observed p-S6k1 to be greater 
when strength training was conducted following endurance exercise than strength training alone. Not only did the authors allocate a $6 \mathrm{~h}$ interval between endurance and strength training, but also provided participants with a meal (containing; $2.02 \mathrm{~g} \mathrm{CHO} \cdot \mathrm{kg}^{-1} \mathrm{bw}, 0.62 \mathrm{~g}$ protein $\cdot \mathrm{kg}^{-}$ ${ }^{1} \mathrm{bw}$ and $0.49 \mathrm{fat} \cdot \mathrm{kg}^{-1} \mathrm{bw}$ ) and a commercially available energy drink following endurance exercise/prior to the strength training protocol. This resulted in glycogen levels being similar between trials involving endurance prior to strength training and strength training in isolation, and may account for the augmenting effect of prior cycling exercise on the anabolic responses to strength training. These findings indicate that if strength training is to be performed subsequent to endurance training on the same day, or within the same session, a feeding strategy between exercise bouts may prevent "unfavourable" signalling responses for strength training related adaptation. In addition, Lundberg et al. ${ }^{27}$ allowed $6 \mathrm{~h}$ recovery between strength and endurance training. These data elude to residual fatigue due to close proximity of strength and endurance training resulting in differing anabolic signalling associated with concurrent training. This may explain why previous studies involving short time periods between strength and endurance training 16,17 observed attuenated anabolic signalling. Additionally, research has demonstrated that the inhibition of strength development within a concurrent regimen may be avoided if sufficient recovery periods $(6-8 \mathrm{~h})$ are allowed between strength and endurance training ${ }^{33}$. These findings have implications for periodized programming, specifically if hypertrophy is the priority of a particular macro/micro cycle. In this scenario endurance and strength type training should be isolated from each other to ensure that adequate recovery time is allowed to facilitate "optimal" anabolic responses.

Strength training performance in the present study was similar between conditions, as the preceding bout of endurance training had no effect on participant's ability to maintain the required training intensity. This is contrary to previous research that has indicated strengthtraining quality and quantity is decremented when performed after endurance training ${ }^{33-37}$. This may indicate the endurance training protocol employed in the present study was of insufficient volume and intensity to induce any noteworthy fatigue and impair subsequent strength training performance. A similar protocol was employed by Coffey et al. ${ }^{17}$ (30 min cycling at a power output that elicited $\sim 70 \%$ of $\left.\dot{V} \mathrm{O}_{2 \text { peak }}\right)$. Whilst these authors did not report strength-training performance it was observed that p-AMPK did not significantly increase from baseline at any point of the experimental protocol (strength then endurance training or vice versa). These data may suggest that those seeking to further investigate the molecular adaptations to strength and endurance training should employ an endurance protocol that does not consist of $30 \mathrm{~min}$ cycling at $\sim 70 \% \dot{V} O_{2 \text { peak }} / \mathrm{p} \dot{V} O_{2 \max }$, as limited phosphorylation of the relevant analysed signalling protocols occur following this protocol. Perhaps high intensity interval exercise should be considered as an alternative due to its potency to upregulate relevant signalling cascades $16,17,38$.

The present study is not without limitations. Firstly, signalling responses were only assessed immediately post and $1 \mathrm{~h}$ post exercise, as such it is possible that differing signalling responses would had been observed if muscle tissue collection took place at $\geq 3 \mathrm{~h}$ post exercise, as others have observed difference signalling responses at this time point ${ }^{17}$. It may also be reasonable to suggest that the (although small) protein bolus provided to the trail may have influenced the signalling responses to the exercise loadings. Additionally, fractional protein synthesis rate was not directly measured but merely anabolic signalling as a proxy of protein synthesis. As such future research should perhaps employ a similar design to that of the present study but extend the post exercise period in which signalling was assessed. Furthermore a direct assessment of total protein synthesis would provide a more robust representation of the influence of concurrent training sequencing on anabolic responses. 


\section{Conclusions}

This study investigated the molecular responses to acute diverse contractile activity in a fed state. Data indicate that ST, ST-END and END-ST elicited similar responses of both the mTOR and AMPK networks. As such, data presented in the study indicate that when performed in close proximity, the order of strength and endurance training is inconsequential to anabolic and energy modulating signalling when performed in a fed state. 


\section{Acknowledgements}

Nil.

\section{Funding statement}

Nil relevant.

\section{Competing interests}

The authors have no real or perceived conflict of interest in respect of this manuscript. 


\section{References}

1. Baar K, Esser K. Phosphorylation of p70S6kcorrelates with increased skeletal muscle mass following resistance exercise. American Journal of Physiology-Cell Physiology. 1999;276(1):120-127.

2. Nader GA, Esser KA. Intracellular signaling specificity in skeletal muscle in response to different modes of exercise. Journal of Applied Physiology. 2001;90(5):1936-1942.

3. Bolster DR, Kubica N, Crozier SJ, et al. Immediate response of mammalian target of rapamycin (mTOR)-mediated signalling following acute resistance exercise in rat skeletal muscle. The Journal of Physiology. 2003;553(1):213-220.

4. Koopman R, Zorenc AH, Gransier RJ, Cameron-Smith D, van Loon LJ. Increase in S6K1 phosphorylation in human skeletal muscle following resistance exercise occurs mainly in type II muscle fibers. American Journal of Physiology-Endocrinology And Metabolism. 2006;290(6):1245-1252.

5. Atherton PJ, Babraj J, Smith K, Singh J, Rennie MJ, Wackerhage H. Selective activation of AMPK-PGC-1 $\alpha$ or PKB-TSC2-mTOR signaling can explain specific adaptive responses to endurance or resistance training-like electrical muscle stimulation. The FASEB Journal. 2005;19(7):786-788.

6. Baar K. Training for endurance and strength: lessons from cell signaling. Medicine \& Science in Sports \& Exercise. 2006;38(11):1939-1944.

7. Nader GA. Concurrent strength and endurance training: from molecules to man. Medicine \& Science in Sports \& Exercise. 2006;38(11):1965-1970.

8. Baar K. The signaling underlying FITness. Applied Physiology, Nutrition, and Metabolism. 2009;34(3):411-419.

9. Thomson DM, Fick CA, Gordon SE. AMPK activation attenuates S6K1, 4E-BP1, and eEF2 signaling responses to high-frequency electrically stimulated skeletal muscle contractions. Journal of Applied Physiology. 2008;104(3):625-632.

10. Inoki K, Zhu T, Guan K-L. TSC2 mediates cellular energy response to control cell growth and survival. Cell. 2003;115(5):577-590.

11. Mounier R, Lantier L, Leclerc J, Sotiropoulos A, Foretz M, Viollet B. Antagonistic control of muscle cell size by AMPK and mTORC1. Cell Cycle. 2011;10(16):2640-2646.

12. Wilkinson SB, Phillips SM, Atherton PJ, et al. Differential effects of resistance and endurance exercise in the fed state on signalling molecule phosphorylation and protein synthesis in human muscle. The Journal of Physiology. 2008;586(15):3701-3717.

13. Vissing K, McGee S, Farup J, Kjølhede T, Vendelbo M, Jessen N. Differentiated mTOR but not AMPK signaling after strength vs endurance exercise in training-accustomed individuals. Scandinavian Journal of Medicine \& Science in Sports. 2013;23(3):355-366.

14. Apró W, Wang L, Pontén M, Blomstrand E, Sahlin K. Resistance exercise induced mTORC1 signalling is not impaired by subsequent endurance exercise in human skeletal muscle. American Journal of Physiology-Endocrinology And Metabolism. 2013;0.1152/ajpendo.00091.2013.

15. Camera DM, Edge J, Short MJ, Hawley JA, Coffey VG. Early time course of Akt phosphorylation after endurance and resistance exercise. Medicine \& Science in Sport \& Exercise. 2010;42(10):1843-1852.

16. Coffey VG, Jemiolo B, Edge J, Garnham AP, Trappe SW, Hawley JA. Effect of consecutive repeated sprint and resistance exercise bouts on acute adaptive responses in human skeletal muscle. American Journal of Physiology-Regulatory, Integrative and Comparative Physiology. 2009;297(5):1441-1451.

17. Coffey VG, Pilegaard H, Garnham AP, O'Brien BJ, Hawley JA. Consecutive bouts of diverse contractile activity alter acute responses in human skeletal muscle. Journal of Applied Physiology. 2009;106(4):1187-1197.

18. Steinberg GR, Watt MJ, McGee SL, et al. Reduced glycogen availability is associated with increased AMPK $\alpha 2$ activity, nuclear AMPK $\alpha 2$ protein abundance, and GLUT4 mRNA expression in contracting human skeletal muscle. Applied Physiology, Nutrition, and Metabolism. 2006;31(3):302-312. 
19. Hawley JA. Molecular responses to strength and endurance training: Are they incompatible? Applied Physiology, Nutrition, and Metabolism. 2009;34(3):355-361.

20. Walshe I, Robson-Ansley P, Gibson ASC, Lawrence C, Thompson KG, Ansley L. The reliability of the IL-6, sIL-6R and sgp130 response to a preloaded time trial. European journal of applied physiology. 2010;110(3):619-625.

21. Rønnestad BR, Hansen EA, Raastad T. High volume of endurance training impairs adaptations to 12 weeks of strength training in well-trained endurance athletes. European Journal of Applied Physiology. 2012;112(4):1457-1466.

22. Beck TW, DeFreitas JM, Stock MS, Dillon MA. Comparison of the muscle activation pattern for the vastus lateralis before and after an 8-week resistance training program. Biomedical Signal Processing and Control. 2010;5(4):264-270.

23. Peterson MD, Rhea MR, Alvar BA. Maximizing strength development in athletes: a metaanalysis to determine the dose-response relationship. The Journal of Strength \& Conditioning Research. 2004;18(2):377-382.

24. Peterson MD, Rhea MR, Alvar BA. Applications of the Dose-Response for Muscular Strength Development: Areview of Meta-Analytic Efficacy and Reliability for Designing Training Prescription. The Journal of Strength \& Conditioning Research. 2005;19(4):950-962.

25. Adam K, Oswald I. Protein synthesis, bodily renewal and the sleep-wake cycle. Clinal Science. 1983;65(6):561-567.

26. Cohen J. Statistical power analysis. Current Directions in Psychological Science. 1992;1(3):98101.

27. Lundberg TR, Fernandez-Gonzalo R, Gustafsson T, Tesch PA. Aerobic exercise alters skeletal muscle molecular responses to resistance exercise. Medicine and science in sports and exercise. 2012;44(9):1680-1688.

28. Fernandez-Gonzalo R, Lundberg TR, Tesch PA. Acute molecular responses in untrained and trained muscle subjected to aerobic and resistance exercise training versus resistance training alone. Acta Physiologica. 2013;209(4):283-294.

29. Kleger G-R, Turgay M, Imoberdorf R, McNurlan MA, Garlick PJ, Ballmer PE. Acute metabolic acidosis decreases muscle protein synthesis but not albumin synthesis in humans. American Journal of Kidney Diseases. 2001;38(6):1199-1207.

30. Caso G, Garlick BA, Casella GA, Sasvary D, Garlick PJ. Acute metabolic acidosis inhibits muscle protein synthesis in rats. American Journal of Physiology-Endocrinology And Metabolism. 2004;287(1):90-96.

31. Creer A, Gallagher P, Slivka D, Jemiolo B, Fink W, Trappe S. Influence of muscle glycogen availability on ERK1/2 and Akt signaling after resistance exercise in human skeletal muscle. Journal of Applied Physiology. 2005;99(3):950-956.

32. Camera DM, West DW, Burd NA, et al. Low muscle glycogen concentration does not suppress the anabolic response to resistance exercise. Journal of Applied Physiology. 2012;113(2):206214.

33. García-Pallarés J, Izquierdo M. Strategies to optimize concurrent training of strength and aerobic fitness for rowing and canoeing. Sports Medicine. 2011;41(4):329-343.

34. Craig BW, Lucas J, Pohlman R, Stelling H. The effects of running, weightlifting and a combination of both on growth hormone release. The Journal of Strength \& Conditioning Research. 1991;5(4):198-206.

35. Leveritt M, Abernethy PJ. Acute effects of high-intensity endurance exercise on subsequent resistance activity. The Journal of Strength \& Conditioning Research. 1999;13(1):47-51.

36. Sporer BC, Wenger HA. Effects of aerobic exercise on strength performance following various periods of recovery. The Journal of Strength \& Conditioning Research. 2003;17(4):638-644.

37. García-Pallarés J, Sánchez-Medina L, Carrasco L, Díaz A, Izquierdo M. Endurance and neuromuscular changes in world-class level kayakers during a periodized training cycle. European Journal of Applied Physiology. 2009;106(4):629-638.

38. Pugh JK, Faulkner SH, Jackson AP, King JA, Nimmo MA. Acute molecular responses to concurrent resistance and high-intensity interval exercise in untrained skeletal muscle. Physiological reports. 2015;3(4):e12364. 


\section{Figures and Tables}

Figure 1. Schematic representation of experimental time line. ST $(n=6)$, ST-END $(n=6$, END-ST $(n=6)$.

Figure 2. Mean training load achieved in the ST $(n=6)$, ST - END $(n=6)$ and END-ST ( $n$ $=6$ ) conditions. ST, strength training alone; ST-END, strength training followed by endurance training; END-ST, endurance followed by strength training. Dashed line indicates required training intensity.

Figure 3. Mean responses of the mTOR signalling network in ST $(n=6)$, ST-END $(n=6)$ and END-ST $(n=6)$ conditions. ST, strength training alone; ST-END, strength training followed by endurance training; END-ST, endurance followed by strength training. (A) p-4EBP1, (B) p-PKB, (C) p-mTOR and (D) p-S6k1. * Significantly greater than pre $(p<0.05)$. ** Significantly greater than post $(p<0.05)$. $\dagger$ Significantly lower than pre $(p<0.05)$.

Figure 4. Mean responses of the AMPK signalling network in the ST $(n=6)$, ST-END $(n=$ $6)$ and END-ST $(n=6)$ conditions. ST, strength training alone; ST-END, strength training followed by endurance training; END-ST, endurance followed by strength training. (A) pACC, (B) p-AMPK, (C) p-eEF2 and (D) p-p38. * Significantly greater than pre $(p<0.05)$.

Figure 5. Representative images of proteins analysed. 